\title{
Multiple Measurements Vectors Compressed Sensing for Doppler Ultrasound Signal Reconstruction
}

\author{
Sulieman M. S. Zobly \\ Dept. of Medical Physics and Instrumentation \\ National Cancer Institute - University of Gezira \\ Wad Medani, Sudan \\ Sulieman16@yahoo.com
}

\author{
Yasir M. Kadah \\ Dept. of System and Biomedical Engineering \\ Faculty of Engineering - Cairo University \\ Cairo, Egypt \\ ymk@,k-space.org
}

\begin{abstract}
Compressed sensing (CS) is a novel framework for reconstruction images and signals. In this work we want to make use of the latest sampling theory multiple measurement vectors (MMV) compressed sensing model, to reconstruct the Doppler ultrasound signal. Compressed sensing theory states that it is possible to reconstruct images or signals from fewer numbers of measurements. In usual CS algorithms, the measurement matrix is vectors so the single measurement vectors (SMV) applied to generate a sparse solution. Instead of using the SMV model we want to make use of the MMV model to generate the sparse solution in this work. Doppler ultrasound is one of the most important imaging techniques. To acquire the images much data were needed, which cause increased in process time and other problems such as increasing heating per unit and increasing the amount of the data that needed for reconstruction. To overcome these problems we proposed data acquisition based on compressed sensing framework. The result shows that the Doppler signal can be reconstructed perfectly by using compressed sensing framework.
\end{abstract}

Index Terms-Compressed sensing, reconstruction, multiple measurements vectors, single measurement vector, Doppler ultrasound.

\section{INTRODUCTION}

Doppler ultrasound is one of the most important tools for measuring and detecting the velocity of moving structures, such as blood flow within the human body. Doppler ultrasound uses reflected sound waves to measure the blood flow through a blood vessel. It helps radiographer to evaluate the blood flow through arteries and veins. Ultrasound imaging application in medical fields has several advantages over other medical imaging modalities. It's used non invasive technique, its cheep, less examination time, movable, the investigation done without any ionizing radiation, capable of forming real time imaging and continuing improvement in image quality [1]. These advantages made ultrasound imaging system is the most widely imaging systems used among others medical imaging equipment.

Data acquisition in Doppler ultrasound relies on transmitting signal continuously to the region of interest and Shannon theory. The theory states that, in order to acquire images or signals perfectly, the sampling theory must be twice the highest frequency in the signal. Thus to acquire Doppler signal a large amount of data were needed in order to get image in real-time with high quality.

The novel theory of compressed sensing (CS) also known under the terminology of compressive sensing, compressive sampling or sparse recovery provides a fundamentally new approach to data acquisition which overcomes the traditional approach of reconstructing signals or images (Shannon sampling theorem) wisdom. It predicts that certain signals or images can be recovered from what was previously believed to be highly incomplete measurements.

Compressed sensing (CS) is a new sampling framework; state that images and signals can be reconstructed by using a few numbers of measurements (what was previously believed to be highly incomplete measurements). CS was first proposed when Donoho published his paper [2] in 2006. CS is the process for acquiring and reconstructing a signal that is supposed to be sparse or compressible. CS is useful in applications where one cannot afford to collect or transmit a lot of measurements such as medical imaging, data compression and data acquisition. There are rapidly growing in application of CS in the field of medical imaging and image processing.

There are two main components of compressed sensing; the sampling strategy and the reconstruction algorithm. Sampling involves measuring a quantity at regular intervals; the concept of sampling in compressed sensing is much more general. Sampling in compressed sensing consists of making random linear projection of the signal into a low dimensional space. The difference between conventional sampling and compressed sensing is that the reconstruction operator is nonlinear. Essentially these select the significant for some sparse representation and then calculate the least square approximation using the associated basis functions.

The main problem in CS is to recover sparse signal $\theta$ from a set of measurements $\delta$, assume that $\delta$ is a linear measurement given by $\delta=\mu \theta$; where $\theta$ is an $N$-by-1, $\delta$ is an $M$-by- 1 vector containing the compressive measurements and $\mu$ is an $M-b y-N$ measurement matrix. The most difficult part of signal reconstruction is to identify the location of the largest 
component in the target signal. The signal reconstruction algorithm must take the $M$ measurement in the vector $\delta$, where $N>>M$. Several methods for recovering sparse $\theta$ from a limited number of measurements have been proposed, such as convex optimization, combinatorial methods and greedy algorithms. Some algorithms have been used for recovering images and signals, such as total variation for MRI image reconstruction and Doppler signal reconstruction via 11 norm, compressive sampling matching pursuit and orthogonal matching pursuit [3 - 6]. In this work we want to make use of the $\mathrm{CS}$ framework based on the multiple measurements vectors algorithm so as to decrease the acquisition time and the number of the data needed for Doppler signal reconstruction.

The rest of the paper is organized as follows: the multiple measurement vectors model and its application described in section two, beside the reconstruction of the Doppler ultrasound signal using MMV model. In section three the experimental result was discussed and shows the effect of increasing and decreasing the number of measurements during the reconstruction. The quality of the images and reconstruction times were also discussed. In the last section the conclusion was presented.

\section{METHODS}

\section{A. Multiple Measurements Vectors Model}

The MMV model is the model assumes that signals and images were matrices and have some sparse structure. This model has founded in array processing [7], non parametric spectrum analysis of time series [8], equalization of sparse communication channels, linear inverse problem [9] and DNA microarray [10].

The MMV model proposed in [11] so as to solve the problem of MMV. Several computation algorithms have been proposed to solve the MMV problem. The OMP is the most suitable algorithm that can find the sparsest solution for MMV [12].

The MMV model, is an $\mathrm{n}$ multiple measurement vector, which can be represented as a measurement matrix $\lambda \in \mathbb{R}^{\mathrm{m} \times \mathrm{n}}$ and the known dictionary $\mu$. the recovered signal represented by matrix $X \in \mathbb{R}^{s \times n}$, our intended in MMV-CS algorithm is to recover the signal $\mathrm{X}$ from the linear equation. The linear equation expressed as follows:

$$
\mu X=\lambda
$$

This problem can be formed as follows:

$$
\min _{x}\|X\|_{2,0}=\text { s.t. } \quad \mu X=\lambda
$$

The $\|X\|_{2,0}$ denotes the sparse matrix, which is measuring the number of no zeros. In other words, the aim is to find the sparse matrix, whose columns possess the same sparsity profile. Considering the noise, the problem becomes as follows:

$$
\min _{x}\|X\|_{2,0}=\text { s.t. } \quad\|\lambda-\mu X\|_{2}<\varepsilon
$$

Where $\varepsilon$ is the amount of noise in the data.

The relation (2) is a combinatorial optimization problem, thus NP-hard, similar to $\ell_{0}$-norm in single measurement matrix (SMV). The matrix $(2,0)$-norm replaced by the matrix $(2,1)$ norm, the relation becomes as follows:

$$
\min _{x}\|X\|_{2,1}=\text { s.t. } \quad \mu X=\lambda
$$

Solving the relation (1) will lead to a good approximation of the signal $X$.

\section{B. Doppler Signal Reconstruction}

The Doppler signal, which is used in this work is a real signal downloaded from H. Torp group website. For Doppler signal reconstruction the measurement matrix $\boldsymbol{\Psi}$ and spares coefficient, which have a problem specific structure, were created. We want to recover a matrix $x$, the Doppler signal in this case, with a few numbers of non-zero component that is, with the MMV recovery algorithm.

In this part we want to show to apply Multiple Measurements Vectors algorithm to the Doppler ultrasound signal recovery problem. Doppler ultrasound signal was sampled randomly and constructed by using CS via MMV algorithms to regenerate a reconstructed Doppler signal, which is used to generate a Doppler ultrasound spectrum using much fewer numbers of measurements $M$.

The Doppler signal, which is used, is a single vector model. The signal rearranged and each column of the measurement represented as a measurement matrix for the Doppler signal, thus the Doppler signal or image formation became a multiple measurement vector.

For Doppler signal reconstruction we try to find the column of $\mu$ most related to the measurements. Then, the algorithm repeats this step by correlating the columns to the signal residual, which is obtained by subtracting the contribution of a partial estimate of the signal from the original measurement vector. The result recovered signal is exactly as the original signal.

\section{RESULTS AND DISCUSSION}

To reconstruct the Doppler signal the Doppler data with the length of 2032, which is downloaded from H. Torp group website was used. A software program written in Matlab (Mathworks, MA) was developed and used to generate both original and reconstructed Doppler spectrogram. Different numbers of measurements were considered for reconstruction. The MMV algorithm was used to perform the Doppler signal reconstruction, which is also is a software program written in Matlab.

Figure 1, shows the recovered Doppler ultrasound spectrum using different number of measurements generated from the 
recovered signal via CS-MMV algorithm. The result shows that the spectrogram was reconstructed perfectly even by using fewer numbers of measurements.
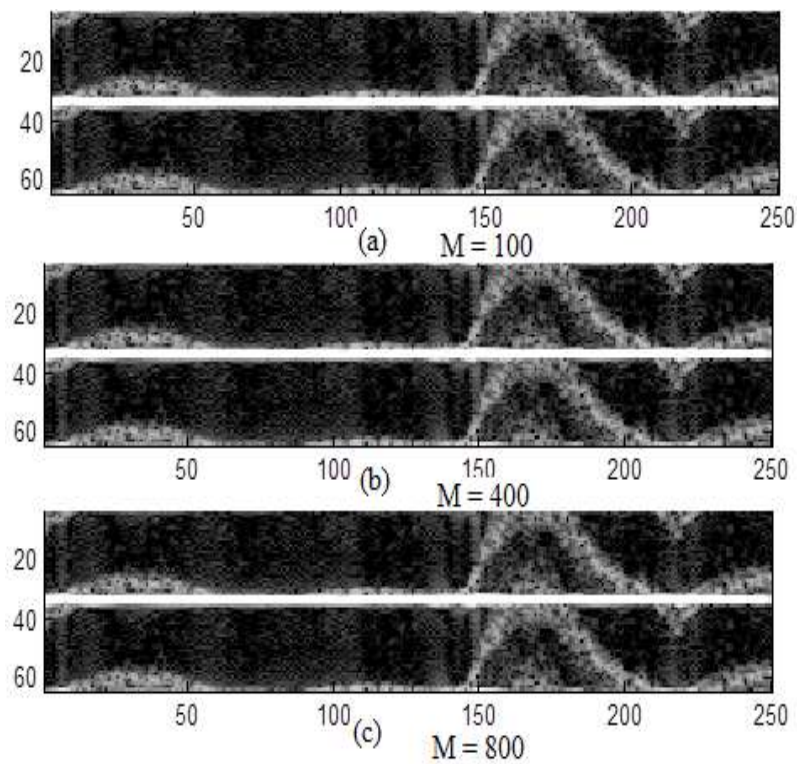

Fig. 1. The recovered Doppler signal using different number of measurements

The error from the recovered images was calculated to compare to the original image; the experimental result shows that the error in the image decreased with increasing the number of measurements. Image reconstructed with higher number of measurements gives error lower than that reconstructed with a few numbers of measurements.

The reconstruction time was calculated at each number of measurements, the result shows that the reconstruction time decreased with the number of measurements, a few numbers of measurements give lower reconstruction time. When higher than $60 \%$ were used there is significantly different in the process time. The result illustrated in figure 2 .

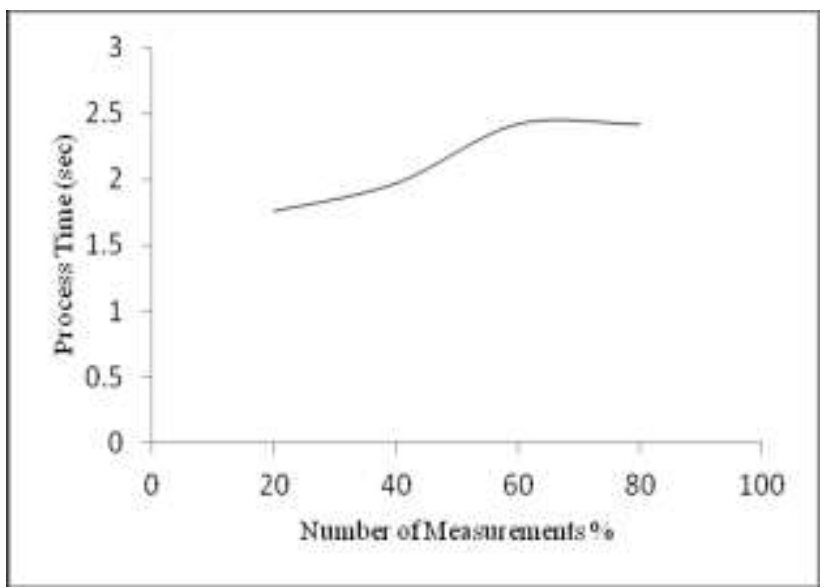

Fig. 2. The number of measurement versus process time

The analysis of the results was performed by calculating the PSNR from each recovered image for different random sample measurements compared to the original spectrogram images, PSNR result shows that the reconstructed image with fewer numbers of measurements has lower PSNR and higher number of measurements gives higher PSNR. This indicates that the quality of the image increased by increasing the number of measurements. Beside the PSNR, the RMSE was used for evaluating the recovered images. A few numbers of measurements give higher RMSE and higher number of measurements gives lower RMSE. Figure 3 shows the relation between the numbers of measurements and RMSE

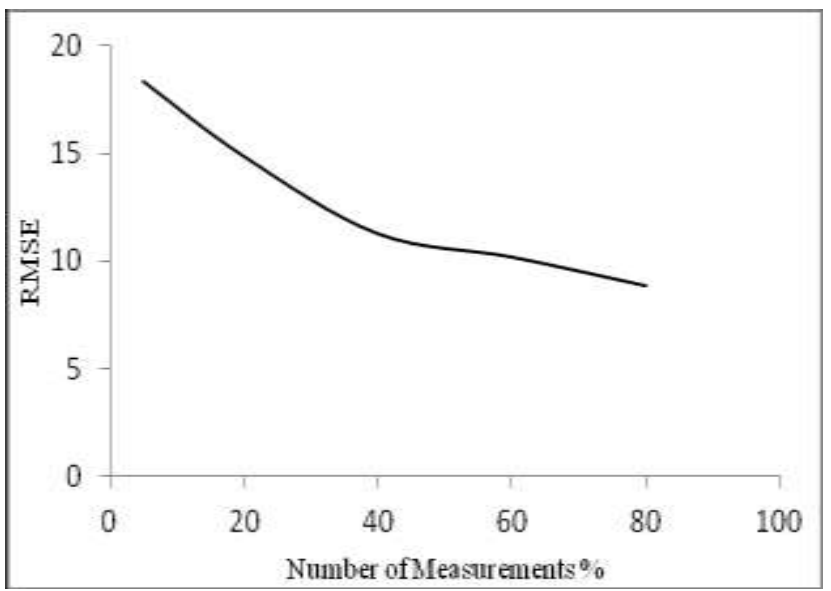

Fig. 3. Number of measurements versus RMSE

\section{CONCLUSION}

Compressed sensing has been applied successfully in different fields such as medical image. The CS framework, used to reconstruct the Doppler ultrasound spectrogram, so as to overcome the current Doppler data acquisition limitation. The reconstruction performed using MMV CS algorithms. It is shown that it is possible to use a very few number of measurements to reconstruct the signal keeping the diagnostic quality intact. This alleviates the major Doppler limitations by reducing the number of acquisitions and eliminating the sampling uniformity constraints. The quality of the recovered image evaluated by using RMSE and PSNR, the result shows that the quality increased by increasing the number of measurements. Also, the recovered time for different number of measurements were calculated. MMV-CS algorithm recovered the Doppler signal with a unique time and good quality.

\section{ACKNOWLEDGMENT}

We would like to thank Professor H. Torpp group for their interesting data.

\section{REFERENCES}

[1] D. Evans, and W. McDicken, Doppler ultrasound: physics, instrumentation and signal processing second ed. John Wiley \& Sons Ltd., New York, 2000.

[2] D. Donoho, "Compressed Sensing", IEEE Trans. Info. Theory, vol. 52, no. 4, pp. 1289 - 1306, 2006. 
[3] M. Lusting, D. Donoho, and J. Pauly, "sparse MRI: The Application of Compressed Sensing for rapid MR Imaging," Magn. Reson. Med., vol. 58, no.6, pp. 1182 - 1195, 2007.

[4] S. Zobly and Y. Kadah, "Compressed Sensing: Doppler Ultrasound Signal Recovery by Using Non-uniform Sampling \& Random Sampling", $28^{\text {th }}$ National Radio Science Conference 2011.

[5] S. Zobly and Y. Kadah, "Novel Doppler Ultrasound Data Acquisition framework based on compressed sensing", International conference on: Advances on biomedical engineering, pp. 137 - 138, 2011.

[6] S. Zobly and Y. Kadah, "Orthogonal Matching Pursuit and Compressive Sampling matching pursuit for Doppler Ultrasound Signal Reconstruction", International Conference in Biomedical Engineering, pp. 52 - 55, 2012.

[7] I. Gorodnitsky and B. Rao, "Sparse Signal Reconstruction from Limited Data using FOCUSS: A re-weighted minimum norm
Algorithm”, IEEE Transaction on Signal Processing, vol. 45, no. 3, pp. 600 - 616, 1997.

[8] P. Stoica and R. Moses, "Introduction to Spectral Analysis", upper Saddle River, NJ: Prentice-Hall, 1997.

[9] S. Cotter, B. Rao, K. Engan and K. Delgado, "Sparse Solution to Linear Inverse Problems with Multiple Measurements Vectors", IEEE Transaction on Signal Processing, vol. 53, no. 7, pp. 2477 $-2488,2005$.

[10] S. Erickson and C. Sabatti, "Empirical Bayes Estimation of A Sparse Vector of Gene Expression Changes", Statistical Applications in Genetics and Molecular Biology, vol. 4, no. 1, pp. 22, 2008.

[11] L. Sun, J Liu and J. Ye, "Efficient Recovery of Jointly Sparse Vectors, In Adv, NIPS, 2009.

[12] H. Lu, X. Long and J. Lv, "A Fast Algorithm for Recovery of Jointly Sparse Vector Based on the Alternating Direction Methods", International Conference on Artificial Intelligence and Statistic, vol. 15, pp. 461- 469, 2011. 